\title{
Impacto Vital del Paso por un COD en la Adolescencia. la Experiencia de Cuatro Adultos de Temuco
}

\author{
The Life Marking Experience of Passing Through a \\ Juvenile Offender's Home During Adolescence: A Recount \\ by Four Adults From Temuco
}

Ricardo Pérez-Luco, Leonardo Lagos, Rodrigo Rozas y Jorge Santibáñez

Resumen

El estudio, de carácter cualitativo, recoge una mirada retrospectiva de las vivencias asociadas a la experiencia de vida de cuatro hombres que en su adolescencia permanecieron internados en el COD de Temuco por problemas de conducta. Para conseguir el objetivo se hizo uso de la técnica de Entrevista en Profundidad, la cual permitió indagar acerca de lo que los participantes consideran más relevante en sus experiencias, usando como tema eje el periodo de la adolescencia. El análisis de los datos se realiza mediante la técnica Análisis de Contenido Jerárquico Ponderado; y su validación, a través de las técnicas de Triangulación y Contrachequeo. Los resultados emergentes del relato de los participantes muestran que el paso por el COD es un hecho determinante en sus vidas, pues dividen su experiencia en un "antes", un "durante" y un "después" de éste; siendo muy relevante en los tres momentos los vínculos establecidas, primero con la familia, segundo con los amigos y "tíos" y tercero con familia, pareja y amigos; son éstas las relaciones que los participantes refieren como detonantes y sostenedoras de su comportamiento adaptativo o desadaptativo posterior. La profundidad de la comprensión lograda permite sostener la hipótesis de que una vinculación afectiva tempranamente dañada o interferida, en la adolescencia se puede consolidar como alteración de la vinculación enmarcada en una "identidad delictual", si el joven no logra su "reparación" mediante el apoyo de figuras sustitutas socialmente adaptadas.

\section{Summary}

This qualitative study, gathers a retrospective glance in the life of four subjects who, during their adolescence, spent time at a home for juvenile offenders (COD), due to their troublesome behaviour. The research objective was pursued by using an indepth interview, which allowed for inquiring about which experiences the

* El presente artículo es el resultado de un estudio realizado durante el año 2003 en la asignatura Práctica de Investigación en Psicología de $4^{\circ}$ año de la Carrera de Psicología de la Universidad de La Frontera, por los actuales psicólogos Leonardo Lagos, Rodrigo Rozas y Jorge Santibáñez, bajo la dirección y supervisión del académico Ricardo Pérez-Luco (e-mail: perezluc@ufro.cl), responsable de esta publicación. 
participants' considered most relevant in their lives, using as a reference axis the period of their adolescence. The analysis of the data was made by means of a Weighed Hierarchic Content Analysis technique; and its validation, was obtained through the techniques of Triangulation and Counterchecking. The emergent pattern of results from these analyses showed that their passage through the COD was a keynote fact in their lives, because they divided their life experiences in a "before", "during" and "after", their being there; being of paramount importance at each of these three periods, the bonds they established, first with the family, second with the friends and "uncles" at the institution, and third with their own family, pairs and friends. These are the relationships the participants refer to as triggering factors for initiating and / or maintaining their later adaptive or maladaptive behaviour. The depth of the obtained understanding allows us to sustain the hypothesis that a damaged or interfered affective bond during the adolescent period, can possibly become consolidated as an alteration of bonding embedded in "criminal identity", if the youngster does not reach "reparation" by means of the support of socially adapted surrogate significant others.

\section{Antecedentes}

El fenómeno de la delincuencia juvenil es una realidad patente en nuestra sociedad con innegable impacto en la vida cotidiana. A diario observamos en diferentes medios de comunicación referencias a gran cantidad de delitos, muchos de ellos graves y en su mayoría cometidos por menores de 18 años, de ahí la importancia de profundizar en la comprensión del fenómeno, aportando datos complementarios a los de incidencia y caracterización descriptiva, regularmente difundidos, a fin de proponer intervenciones cada vez más pertinentes para este grupo etáreo, más aún en el marco de la implementación de la LEY DE RESPONSABILIDAD PENAL JUVENIL.

El término delincuencia juvenil se usa para referir a menores de 18 años que cometen delitos. En la mayor parte de los países, la delincuencia juvenil recibe un tratamiento judicial distinto al que reciben los mayores de edad en un intento de maximizar su rehabilitación. Al igual que ocurre entre los adultos, la mayor parte de los delitos cometidos no se detectan, por lo que cabría diferenciar entre la delincuencia juvenil detecta- da que queda reflejada en las cifras oficiales de incidencia y la delincuencia juvenil sumergida o no detectada. Además, hay que indicar que los patrones delictivos de aquellos jóvenes detectados difieren de los no detectados, al presentar mayor frecuencia y gravedad (Cooper, 1994; Vergara, 1996).

En Chile se observa que la mayoría de los delitos son cometidos por jóvenes, pues se registra que un $64 \%$ de los aprehendidos tiene entre 10 y 24 años y los hurtos y robos en su mayoría son realizados por adolescentes entre 15 y 19 años (Paz Ciudadana, 2003). Si bien las estadísticas aportan información muy interesante, los estudios sociológicos y psicológicos son imprescindibles para comprender mejor las causas y dinámica de este fenómeno.

Cabe preguntarse ¿por qué una mayor delincuencia durante la adolescencia? Podría tener relación con la naturaleza de la etapa; con que cede el control paterno sin que aun se asuman las responsabilidades de adulto; con la influencia de los pares; o también como consecuencia de la fabulación propia de la edad (Ávalos, 1990). Asumiendo el enfoque ecosistémico, no puede hablarse de una causa de la delincuencia, sino de diversos facto- 
res que actúan simultánea o sucesivamente y que interactúan entre sí. En algunos casos podrán tener más presencia los factores psicológicos y en otros los sociales. En este sentido algunos autores diferencian entre el delincuente sociológico, en el que el comportamiento antisocial sería parte integral del estilo de vida propio de un grupo perteneciente a sectores pobres; y el delincuente individual, en el que predominan factores individuales y familiares (Llanos y Sinclair, 1996).

Los estudios en adolescentes infractores dan cuenta de un autoconcepto pobre y baja autoestima, asociado a malas relaciones familiares y fracasos escolares. Hombres y mujeres suelen tener bajo desarrollo del razonamiento moral situándose en un nivel preconvencional. Se muestran desafiantes con la autoridad, resentidos, agresivos y hostiles; presentan una importante falta de autocontrol, probablemente como consecuencia de una pobre socialización; en la escuela se distraen fácilmente, son poco persistentes en sus tareas, con malos hábitos de trabajo, problemas de conducta, bajo rendimiento y un alto grado de ausentismo, que genera más tiempo libre sin supervisión (Ávalos, 1990, Fuenzalida, 1996).

Abundantes investigaciones encuentran una clara relación entre hogares rotos (por separación, divorcio o fallecimiento) o desestructurados y la conflictividad y delincuencia juvenil. La ausencia del padre aparece como un factor muy relevante, ya que puede llevar a una personalidad masculina sobrecompensadora y agresiva que se relaciona con la conducta agresiva. También se asocia a ambientes más permisivos por ausencia de control y pautas disciplinarias erráticas, pues se ha observado que los padres de adolescentes que delinquen suelen ser o indiferentes o muy punitivos y estrictos, con hostilidad y rechazo hacia el niño, lo que genera mayor agresividad. También se observan débiles vínculos emocionales y una actitud negativa hacia los padres (Fischman, 1989; Rodríguez, 1989; Llanos y Sinclair, 1996; Valverde, 1996; Zambrano, 2001).

Muchos autores coinciden en señalar que en las áreas urbanas con mayores niveles de población, la pobreza y la marginación a partir de la infancia imposibilitarían la incorporación de este sector a la sociedad global. Esta situación generaría, en parte de esta población, la búsqueda de alternativas viables ilegales para la sobrevivencia. De acuerdo a esto, la exclusión que viven las familias en extrema pobreza les impide acceder a recursos básicos para su adecuado desenvolvimiento, obligándolas a desarrollar formas de adaptación a la realidad que implican la aparición de problemas como frustración, violencia doméstica, alteraciones de la comunicación, desarme y rearme de parejas, ingreso temprano al mundo laboral, etc. (Pérez-Luco, 1994; Zambrano, 2001).

Las menores oportunidades que ofrece el vivir en una situación de pobreza es un reiterado factor de riesgo, sin embargo, esta situación está cambiando por el surgimiento de nuevos tipos delictivos. Datos recientes con cifras de delincuencia no detectada indican una débil relación entre clase social y actividad delictiva, ya que los jóvenes de clase media reconocen similar cantidad de delitos que los de clase baja, aunque menos graves. Una gran diferencia es que los adolescentes de clase media y acomodada cuando cometen un delito no son arrestados y denunciados o lo son con menos frecuencia, debido a la influencia posible derivada de la posición social que ocupan (Valverde, 1996).

Esto no quiere decir que todos quienes viven en condiciones de pobreza presenten estos problemas, ni que estas dificultades sean exclusivas de este sector. Aquí se propone que la emergencia de algunos trastornos psicosociales puede ser en muchos casos un intento de adaptación a circunstancias de vida que no proporcionan oportunidades 
para un desarrollo pleno de las potencialidades (Pérez-Luco, 1994; Zambrano, 2001).

El surgimiento de problemas psicosociales se relaciona con las posibilidades que quienes pertenecen a un determinado grupo social tienen de ejercer control sobre el medio ambiente. La dificultad para ejercer control en cuestiones tan vitales como satisfacer las necesidades básicas para la subsistencia, genera consecuencias en las personas como la sensación de que los eventos de la vida son definidos y resueltos por otros, perdiéndose así la fe respecto de que las condiciones actuales o futuras pueden ser modificadas por la propia acción y por tanto, la motivación para mejorar las condiciones actuales. Esta falta de poder objetivo de las personas sobre su situación vital se perpetúa en la medida que los individuos se paralizan y sólo se movilizan en torno a la sobrevivencia cotidiana (Zambrano, 2001).

Las condiciones de vida precaria, en lo material y lo afectivo, imposibilitan a los niños que allí se desarrollan el aprender oportunamente normas, formas de relación, habilidades y competencias que les permitan integrarse adecuadamente a la vida social; considerando la noción de «adecuadamente» para hacer referencia a los estándares sociales establecidos como deseables para la convivencia social. Estos vacíos educativos tienen implicancias en la construcción de identidad, ya que el niño, al tener dificultades para presentar las conductas esperadas para su edad y para interactuar apropiadamente con los otros, experimentará la tensión de no responder a las expectativas sociales, redundando a nivel psicológico en una baja autoestima, sentimientos de frustración y surgimiento de mecanismos psicológicos que permitan compensar las dificultades experimentadas en la relación con los otros (Ávalos, 1990; Conejeros y Torres, 1992; Zambrano, 2001).

De acuerdo a Jesús Valverde (1996), la inadaptación social es un proceso que surge a propósito de condiciones adversas de vida que imposibilita el desarrollo de cierto tipo de recursos que son exigidos por el medio social. Así, la inadaptación social resulta ser una forma de adaptación (difícil) a condiciones de vida que no satisfacen las necesidades de los niños, quienes deben desarrollar estrategias alternativas para responder a las múltiples demandas de un medio que no ofrece oportunidades. Los adolescentes en este contexto enfrentan circunstancias muy adversas, debiendo hacer uso de sus mejores recursos para lograr una adaptación exitosa, incluso se podría sugerir la delincuencia como una respuesta resiliente en un entorno deprivado y dañador (Pérez-Luco, Alarcón y Zambrano, 2004)

La dinámica familiar se ha estudiado regularmente como factor protector tanto como factor de riesgo. La capacidad de resolver conflictos y desarrollar estrategias adaptativas se activa en la evolución familiar, pero en este entorno regularmente se describe a las familias como disfuncionales o en riesgo, pues en vez de favorecer el desarrollo integral de sus miembros y otorgar protección especial a aquellos que están en desarrollo, provocan daño y experiencias traumáticas por conflicto interno, negligencia o incapacidad, al no lograr cumplir las mínimas funciones que la definen, pese a los múltiples apoyos externos recibidos (Alarcón y Troncoso, 2001; Oñate y Calfuquir, 1998).

La capacidad de recurrir y usar redes para el afrontamiento de los problemas familiares señala que las familias en riesgo psicosocial recurren y se perciben a sí mismas con menos redes formales, informales y familiares; esto demuestra la autopercepción de menor apoyo social en los ámbitos instrumentales y afectivos. Así también es posible señalar que la percepción de los padres respecto a la vinculación afectiva, cohesión, flexibilidad y comunicación entre padres e hijos, aparecen significativamente disminuidas en las familias de riesgo (Oñate y Calfuquir, 1998). 
Estas familias, por lo general, son asistidas por un conjunto de instituciones que con diversas miradas teóricas y metodológicas desean promover su desarrollo y mejorar su calidad de vida. En este sentido se puede considerar el concepto de familias multiasistidas, en las cuales las instituciones van observando variadas características familiares y trabajando con ellas al mismo tiempo que las familias van conociendo los modos de funcionamiento de cada institución, estableciendo relaciones particulares con cada una de ellas (Martínez, Neculhueque, Oliveros, y Soto, 2003).

Una dinámica relevante se observa en la intervención de maltrato infantil grave. Son familias en que prevalece la violencia, con límites rígidos, con escasa red de apoyo social y tendencia a aislarse de otros contactos sociales; suele observarse estrés familiar asociado a problemas psicosociales como cesantía del proveedor, separación de la pareja o alcoholismo o drogadicción de uno o ambos padres; y un número importante de ellas vive en condición de extrema pobreza y con fuertes niveles de deprivación en diversas áreas de su desarrollo (Alarcón y Troncoso, 2001).

Por su parte, los niños maltratados, mayoritariamente no se perciben a sí mismos en calidad de víctimas, los golpes aparecen como eventos cotidianos en sus vidas por lo que adquieren cierta dosis de «naturalidad» para ellos. Con frecuencia los padres que maltratan a sus hijos presentan experiencias de carencias afectivas tempranas, abandono o historial de maltrato, siendo a su vez hijos de padres maltratadores. La figura materna aparece pasiva y en ocasiones con problemas psicológicos, requiriendo resolver conflictos personales previo a avanzar en la generación de recursos que eviten el maltrato infantil. La figura paterna con frecuencia presenta dificultades en el control de impulsos, mostrando escasa tolerancia y sin habilidades para atender las demandas de sus hijos; y ambos padres (o figuras sustitutas) presen- tan dificultades para ejercer la autoridad de modo consistente (Barudy, 2001). En paralelo, al estudiar las familias en que ocurre el abuso sexual intrafamiliar, se detectan límites familiares difusos con alteración jerárquica y parentalización de los niños; y con madres que a menudo presentan inestabilidad emocional, por depresión o estrés y convivencias o parejas múltiples (Alarcón y Troncoso, 2001).

En las familias marginales encontramos que los padres son individuos que en su gran mayoría no han completado su desarrollo emocional, ya que han debido atravesar diversas experiencias límites, como la temprana búsqueda de alternativas de subsistencia, la falta de afecto y de apoyo de sus padres, la pérdida o ausencia de éstos y también la represión directa de los servicios policiales; lo que incluye detenciones, interrogatorios, encierros, allanamientos e incluso tortura (Pérez-Luco y Alarcón, 1992).

Las experiencias vivenciadas por los progenitores, dada su condición de sobrevivencia, se van acumulando a lo largo de su historia y la mayoría de las veces sobrepasan los recursos psicológicos y sociales que poseen, pues al existir una exposición crónica a experiencias límites se favorece la insatisfacción de necesidades afectivas y sociales, las que tienden a actualizarse en los vínculos filiales; generando en los hijos consecuencias psicológicas negativas como dificultad de individuación y presencia de comportamientos autodestructivos (Sepúlveda y Contreras, 1985).

El fracaso del proceso de individuación a temprana edad implica que el niño renuncia a satisfacer sus propias necesidades al establecer sus primeros vínculos afectivos ya que la madre tiende a traspasar su experiencia traumática, sobrecargando al niño con sus propias necesidades y siendo incapaz de reconocer las necesidades del hijo. Este fenómeno se ha descrito como el traspaso de un 
«trauma acumulado», siendo característico de progenitores que han sufrido experiencias traumáticas extremas (Biedermann y Díaz, 1991); los niños pierden el lugar de «centralidad necesaria» para captar el apoyo afectivo en la dinámica familiar, alterándose su proceso de crecimiento y desarrollo (Pérez-Luco y Alarcón, 1992).

Los niños al interior de la familia no sólo se hacen cargo de los traumas acumulados a través de generaciones, sino que al mismo tiempo resultan entrampados entre las demandas contradictorias de los padres, quienes les solicitan ser autosuficientes e independientes y al mismo tiempo no dejar de satisfacer sus necesidades (de los padres). A modo de ejemplo se puede observar cómo «hijos-hombres» tienden a suplir al padre ausente en relación a la subsistencia y las necesidades afectivas de la madre y las «hijas-mujeres», tienden a suplir el rol materno en la relación con sus hermanos. El fracaso ante estas exigencias deriva de la imposibilidad de asumir demandas en sí mismas contradictorias y al mismo tiempo reconocer las propias necesidades potenciales, lo que es particularmente evidente durante la adolescencia (Pérez-Luco y Alarcón, 1992).

Alteraciones de conducta como la delincuencia, el alcoholismo y la prostitución, muchas veces pueden ser una respuesta a demandas familiares que chocan con un entorno aversivo y hostil, generando alienación social. Los niños que delinquen tenderían a percibir el mundo y sus procesos como algo que es y ocurre independientemente de ellos, "miran desde el lado exterior de la vitrina» son espectadores de lo que sucede a su alrededor; esto implica la vivencia de estar ajeno, de no pertenencia, de exclusión, pues lo que hay al otro lado de vidrio no les pertenece, el mundo es lo que es independiente de ellos. Como consecuencia del mirar hacia fuera y verse ajeno, aparece el mirar hacia adentro y no encontrar nada de valor; la percepción de estos niños, es que no importa lo que hagan, las cosas seguirán siendo iguales, el mundo nunca será su mundo y su pobreza será permanente, percepción arraigada en vivencias muy básicas de condicionalidad afectiva, abandono, desprotección y marginación, las que forman parte central de su construcción de identidad (Alarcón, Pérez-Luco y Lucero. 1992).

La percepción del mundo ajeno se asienta en una condición objetiva de amenaza en un modo vivencial característico que se reproduce en los vínculos familiares al incorporar la experiencia de opresión social como pauta de interacción familiar. El niño se desarrolla en el seno de experiencias familiares de desprotección, abandono afectivo, inestabilidad, exigencia temprana de independencia en la satisfacción de necesidades básicas y demanda de contribución a la economía doméstica; experiencias que se ven reforzadas en el paso por las instituciones asistenciales, constituyéndose en patrones de interacción adaptativos a las condiciones de vida. Los niños se vivencian y se autoperciben marginales, inadecuados, malos y desadaptados; su historia reiteradamente les demuestra que no valen nada y su paso por las instituciones no hace más que confirmar esta idea. Además, estos niños se vinculan socialmente a través de los organismos policiales y el sistema judicial, instituciones que reiteradamente les señalan como delincuentes, marginales o excluidos, dándoles el trato que se "merecen". La recurrencia de esta vivencia hace que sea asumida como algo propio que los identifica, lo que puede significar la paradoja de definirse como delincuente para poder tener un espacio que ocupar en la sociedad (Pérez-Luco y Alarcón, 1992).

Se comprende así la aceptación de una identidad delictiva, pues el delinquir, además de proveer de los medios de subsistencia necesarios, se constituye en una buena forma de resolver el conflicto de pertenencia-no pertenencia social, pues se les asigna un rol social (son considerados) y a la vez garantiza el cas- 
tigo buscado por ser malos (Alarcón, PérezLuco y Lucero. 1992). Esto termina de confirmar los preceptos negativos sobre los cuales se ha constituido su identidad. Cierra las posibilidades de cambio lo que produce una fuerte sensación de desamparo y frustración y conduce necesariamente a un vivir centrado en el presente a modo de defensa frente a un futuro ciertamente negativo.

\section{Método}

Participantes: Se usó un muestreo intencionado de casos típicos en base a los criterios de sexo, edad y causal de ingreso al COD. Participaron cuatro adultos de sexo masculino cuyas edades fluctuaron entre los $25 \mathrm{y}$ 30 años, actualmente residentes en la ciudad y diagnosticados en el COD por "problemas de conducta".

Diseño: Dado que lo que se buscaba era la comprensión de la experiencia de los participantes, la investigación fue de tipo descriptivo con un acercamiento fenomenológico, mediante un Diseño de Casos Múltiples.

Técnica: La recolección de los datos se realiza mediante Entrevista en Profundidad (Taylor y Bogdan, 1986), complementada con registro escrito en Nota de Campo y registro magnético en cinta de audio.

Procedimiento: Se solicitó permiso a la Dirección Regional de SENAME para acceder a los a archivos históricos del CTD Alborada de Temuco (ex COD). De los registros disponibles se seleccionaron 12 casos que, siendo adolescentes entre los años 1989 a 1992, estuvieron internos y fueron diagnosticados en dicho centro por la causal antes señalada. Siguiendo los datos registrados en los informes y referencias obtenidas en terreno por informantes claves, se pudo ubicar a seis sujetos en su contexto natural, a quienes se les explicó los fines de la investigación y resguardos éticos propios del estudio. Todos acceden voluntariamente a participar por lo que se fijan nuevos momentos para la realización de las entrevistas, estableciéndose de mutuo acuerdo los sitios y horarios de encuentro (domicilio personal o cafetería del centro de la ciudad), sin embargo, dos no se presentan ni son asequibles posteriormente. A los cuatro entrevistados se les ubica después para hacer la devolución de la entrevista transcrita y realizar el contrachequeo.

\section{Resultados}

Se utilizó el Método de Análisis de Contenido Jerárquico Ponderado (Pérez-Luco, 2003), cuyo objetivo final es extraer de lo reportado por los sujetos (desde la base), una estructura de significados que permita comprender en profundidad el fenómeno estudiado. De este modo, los datos transcritos fueron depurados en fases sucesivas, realizando primero una codificación abierta que permitió la preparación de fichas de codificación estructuradas en parágrafos y el desarrollo de un sistema de categorías jerárquico en forma de árbol o dendograma con tres núcleos centrales de contenido (ver esquema 1).

\section{Esquema 1}

\section{Núcleos centrales de Contenido}

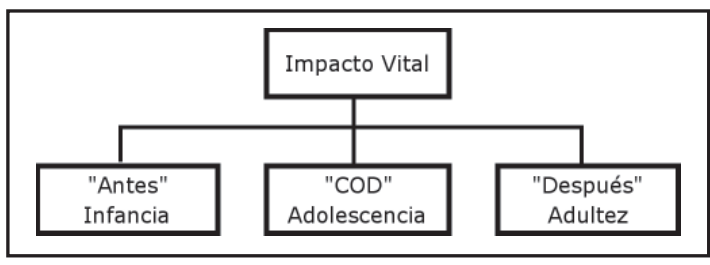

En la siguiente fase, usando los códigos derivados del dendograma, se codificaron las entrevistas en las fichas preparadas, triangulando los resultados, y se estimaron las frecuencias de ocurrencia de cada código para el total de sujetos y por separado para los dos que continuaron delinquiendo y los dos que no lo hicieron a fin de buscar aspectos diferenciales en sus experiencias que hiciesen comprensibles las distintas 
“opciones" (ver tablas 1 y 2). Es relevante señalar que el hecho que dos de los participantes hubiesen continuado robando, con posteriores reclusiones por ello y los otros dos no, resultó así sin necesidad de intencionarlo, aún cuando era un propósito previo el buscar los dos perfiles.
La reducción final de los datos permitió establecer que los cuatro participantes evalúan retrospectivamente que su experiencia infantil previa al ingreso al COD se encontraba saturada de vivencias negativas:

“...mi mami murió cuando tenía 9 años... mi papá trabajaba afuera...

\section{Tabla 1}

Peso Relativo de contenidos en las Categorías Construidas

\begin{tabular}{|cl|c|c|c|c|}
\hline Categoría y código & $\begin{array}{c}\text { Frecuencia } \\
\text { de códigos }\end{array}$ & $\begin{array}{c}\% \text { peso } \\
\text { relativo }\end{array}$ & $\begin{array}{c}\text { Frecuencia } \\
\text { de códigos }\end{array}$ & $\begin{array}{c}\% \text { peso } \\
\text { relativo }\end{array}$ \\
\hline $\mathbf{1}$ & Antes: Infancia & & & $\mathbf{7 9}$ & $\mathbf{2 2 , 5}$ \\
\hline 1.1 & Vínculos familiares & 39 & 49,3 & & \\
\hline 1.2 & Amigos & 15 & 18,9 & & \\
\hline 1.3 & Experiencia escolar & 25 & 31,6 & $\mathbf{1 0 4}$ & $\mathbf{2 9}, \mathbf{6}$ \\
\hline $\mathbf{2}$ & COD: Adolescencia & & & & \\
\hline 2.1 & Percepciones & 16 & 15,3 & & \\
\hline 2.2 & Sentimientos & 77 & 74,0 & & \\
\hline 2.3 & Relaciones & 11 & 10,5 & & \\
\hline 3 & Después: Adultez & & & & \\
\hline 3.1 & Adaptación Social & 62 & 36,9 & & $\mathbf{1 0 0}$ \\
\hline 3.2 & Vínculos Afectivos & 56 & 33,3 & & \\
\hline 3.3 & Proyecto vital & 50 & 29,7 & & \\
\hline Total & & & & \\
\hline
\end{tabular}

yo lo pasaba en la calle... me sentía solo... empecé a robar p'a comer... le hacía al neoprén..."

“...habian problemas en mi casa con mi mami y mi papi... mi papá era bueno p'al frasco... nos sacaba la chucha... me arranqué de la casa a los 8 años... dormía en la calle... empecé a meterme en otras huevás como el neoprén... sólo me entregué a los pacos..."
El paso por el COD para todos resultó una experiencia vital determinante que marca un punto de inflexión en sus historias, pero vivenciada de modo diferencial por quienes continuaron delinquiendo y quienes no lo hicieron:

“...fue fulero... en vez de bien fue p'a más malo... te hacían pelear... era igual que un regimiento... yo vicuando se violaron a un cabro... ahíme hice de hartos amigos que estaban en la movía... no me rehabilité..." 
“...empecé a recapacitar... los tíos se preocupaban por mí... me enseñaron a valorar lo que era yo... me decían lo que tenia que hacer... pude trabajar en la cocina y seguir estudiando... me tenían confianza... me sirvió harto... yo cambié..."
Sin embargo, las carencias han persistido en todos, aún cuando dos refieren haber optado por mejorar, dedicándose a estudiar y trabajar y dos se mantuvieron en el "ambien$t^{\prime \prime}$, con nuevos ingresos al COD y periodos de reclusión posterior.

\section{Tabla 2}

Diferencias de énfasis entre perfiles distintos

\begin{tabular}{|c|c|c|c|c|}
\hline \multirow[b]{2}{*}{ Categoría y código } & \multicolumn{2}{|c|}{$\begin{array}{c}\text { Sujetos } \\
\text { con reclusión por robo }\end{array}$} & \multicolumn{2}{|c|}{$\begin{array}{c}\text { Sujetos } \\
\text { con adaptación laboral }\end{array}$} \\
\hline & $\begin{array}{l}\text { Frecuencia } \\
\text { de códigos }\end{array}$ & $\begin{array}{l}\% \text { peso } \\
\text { relativo }\end{array}$ & $\begin{array}{l}\text { Frecuencia } \\
\text { de códigos }\end{array}$ & $\begin{array}{l}\% \text { peso } \\
\text { relativo }\end{array}$ \\
\hline 1 Antes: Infancia & 43 & 25,74 & 36 & 19,5 \\
\hline 3.4 Vínculos familiares & 17 & 39,53 & 22 & 61,1 \\
\hline 3.5 Amigos & 12 & 27,90 & 3 & 8,3 \\
\hline 3.6 Experiencia escolar & 14 & 32,55 & 11 & 30,6 \\
\hline 4 COD: Adolescencia & 38 & 22,75 & 66 & 35,8 \\
\hline 4.1 Percepciones & 8 & 21,05 & 8 & 12,1 \\
\hline 4.2 Sentimientos & 19 & 50,00 & 52 & 78,9 \\
\hline 4.3 Relaciones & 11 & 28,94 & 6 & 9,1 \\
\hline 5 Después: Adultez & 86 & 51,49 & 82 & 44,5 \\
\hline 5.1 Adaptación Social & 41 & 47,69 & 49 & 59,8 \\
\hline 5.2 Vínculos Afectivos & 28 & 32,55 & 22 & 26,8 \\
\hline 5.3 Proyecto vital & 17 & 19,76 & 11 & 13,4 \\
\hline Total & & 167 & & 184 \\
\hline
\end{tabular}


En las experiencias familiares previas (ver esquema 2), aparecen con mayor frecuencia los vínculos conflictivos en especial con la figura paterna (padre biológico o padrastro), derivados del alcoholismo, la agresión y despreocupación de éste.

\section{Esquema 2}

Categorías de contenidos significativos de la experiencia previa a la internación

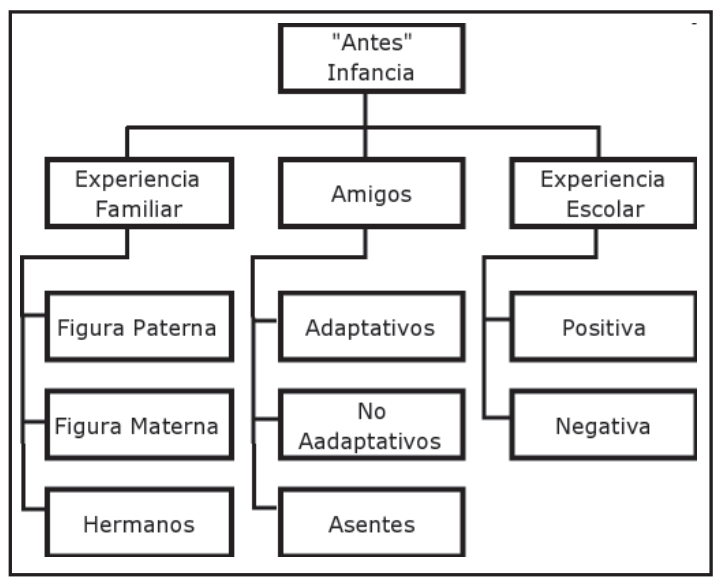

Los vínculos positivos están referidos principalmente a la figura materna como fuente de afecto, luego hermanos y abuelos. Los amigos de la infancia, frecuentemente presentan conductas desadaptativas como consumo de alcohol y drogas y realización de hurtos. El medio escolar, es recordado con desagrado, por rechazo de sus pares y profesores, derivando esto en un abandono temprano; sin embargo, quienes abandonan la carrera delictiva recuerdan como figuras significativas a algunos profesores que los apoyaron, pero a ningún compañero. En todos los casos el primer ingreso al COD fue por medida de protección derivada de vagancia y hurto, algunos presentaban además inhalación de solventes y / o robo y todos presentaron al menos un ingreso posterior.

La experiencia de internación en el COD presenta una fuerte significación afectiva, siendo de tal intensidad las vivencias disfóricas que en el relato de todos los parti- cipantes reaparecen tan vívidas cómo si recién hubiese ocurrido (ver esquema 3). En este sentido los sentimientos de engaño, impotencia y desamparo son tan recurrentes como la percepción de encierro; además, la intensidad de las relaciones establecidas contribuye a fortalecer los sentimientos negativos, en especial por la evaluación de un trato abusivo por parte de los funcionarios (maltrato, castigo injusto, trabajos forzados e indolencia frente al abuso realizado por los más grandes hacia los chicos, incluido el abuso sexual). Sin embargo, también aparece, aunque en menor medida, una evaluación positiva de la experiencia, asociada a la vivencia de acogida por parte de algunos de los funcionarios y profesionales, lo que permitía garantías de mejor trato. Las relaciones entre pares son referidas mayoritariamente conflictivas, de rivalidad, abuso y rechazo, sin embargo, algunos expresan haber experimentado un gran compañerismo y sentimientos de apoyo, respeto y lealtad entre algunos de los internos.

\section{Esquema 3}

\section{Categorías de contenidos significativos de la experiencia de internación}

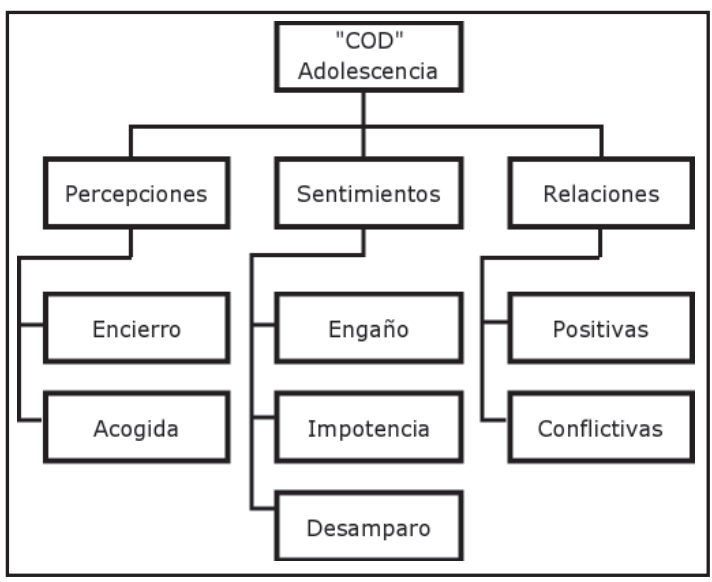

Durante este periodo los participantes refieren un importante distanciamiento familiar lo que los afecta negativamente, pues el contacto se limita a las visitas, en algunos 
casos regulares y en otros muy irregulares, de sus madres y hermanos y raras veces de sus padres; durante las visitas reciben el apoyo y contención que necesitaban, pero luego de ellas relatan sentir una mayor soledad e incluso abandono. Por último, un aspecto muy relevante de esta etapa, transversal a la experiencia, pues surge como evaluación retrospectiva, es la autoimagen individual construida, la que se conceptualiza con base en la identificación delictual o no delictual, guiando los comportamientos dentro del centro, tanto como la identificación con los distintos grupos de internos y con en el contexto del COD.

Mirado desde el momento actual, la experiencia se evalúa de modo ambivalente. Fue muy positiva por cuanto les produjo aprendizajes significativos respecto de sí mismos y de la sociedad ("... aprendí cómo son las cosas... lo que tengo que hacer para vivir... lo que valgo... aprendí a hacerme respetar..."), aprendieron a valorar a la familia la libertad y a sí mismos. Pero fue más negativa por la privación de libertad, la lejanía de los seres queridos, la privación de estudios y de oportunidades personales y en especial por la estigmatización vivida, lo que ha repercutido de modo determinante en su desenvolvimiento posterior.

El egreso fue muy difícil, todos presentaron comportamientos desadaptativos consumo excesivo de alcohol, consumo de drogas y conflictos interpersonales (ver esquema 4). Dos de ellos continuaron delinquiend y y fueron posteriormente recluidos y condenados por ello y actualmente trabajan aunque de manera inestable; los otros dos, si bien confiesan haber cometido pequeños hurtos no se asumen delincuentes, pues no han sido recluidos y han permanecido trabajando.

\section{Esquema 4}

Categorías de contenidos significativos de la experiencia posterior a la internación

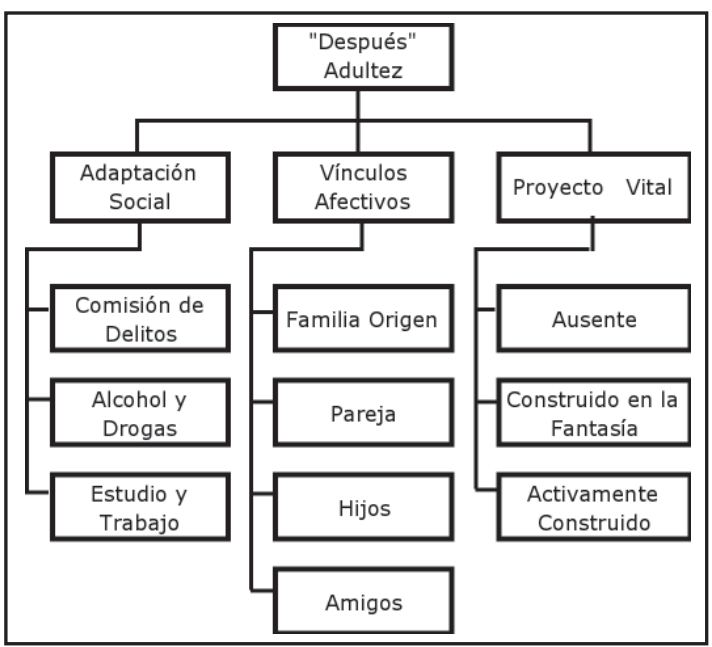

Para todos, el trabajo es un medio de seguridad, que permite la mantención familiar y aunque lo ven obligatorio, brinda autosuficiencia en especial para alimentarse. Sin embargo, sus experiencias laborales han sido irregulares, poco gratificantes o insatisfactorias.

En el ámbito de los vínculos afectivos, pese a observarse diferentes trayectorias, ninguno se manifiesta satisfecho. Los vínculos sostenidos de manera más estable han sido en todos los casos con su familia de origen, especialmente con la madre, vínculo evaluado como el más significativo, salvo en un caso cuya madre fallece muy tempranamente y la figura de referencia pasa a ser el abuelo, quien ha estado siempre presente como figura de identificación con un comportamiento socialmente adaptativo.

En los vínculos de pareja y formación de la propia familia es donde se revela mayor frustración. Si bien todos han tenido pareja y tres de ellos hijos, sólo uno mantiene actualmente una convivencia estable, mostrando interés por sus hijos, los demás no tienen pareja estable y si la tuvieron les duró poco. Sobre los hijos, en general no hablan bien, se encuentran distanciados y uno de ellos no tiene ningún contacto, 
además los dos que han delinquido se asumen malos modelos con los hijos.

Por último, en relación a los amigos, los vínculos se han ido perdiendo, limitándose casi exclusivamente a encuentros puntuales para "alguna movida".

A futuro, todos los participantes se proyectan de modo ambiguo o vago con ausencia de planificación. Sus metas personales son convencionales (familia, pareja, hijos, trabajo, autosuficiencia y continuar estudios) y los sueños poco realistas como la obtención de una casa propia y sacarse el «kino», siendo esto último lo más anhelado por todos.

Conceptualmente, los contenidos se estructuran jerárquicamente para dar respuesta a la pregunta de investigación sobre el impacto vital que tuvo el paso por el COD en la adolescencia, presentando cuatro niveles diferentes: En el nivel más abstracto la respuesta se resume en una experiencia determinante de las historias individuales posteriores, al constituirse en un hito que marca las opciones personales y la definición de identidad en la adolescencia. En el segundo nivel la respuesta se diferencia en dos opciones la no pertenencia ("...ese no era mi ambiente... yo era diferente... no era lo que quería... los tíos me ayudaron a cambiar") y la pertenencia ("... ahí encontré amigos de verdad... era como mi casa... aprendí a defenderme... me hice hombre..."), lo que resulta determinante en la constitución de una identidad delictual. En un tercer nivel surge la vinculación afectiva como elemento articulador de una identidad socialmente adaptada o desadaptada emergiendo las figuras masculinas adultas y el grupo de pares como los referentes más significativos. Por último, en el cuarto nivel aparece la gratificación afectiva brindada por la madre o la pareja, como elemento reparador; versus la frustración afectiva familiar (familia de origen, relación de pareja y paternidad) como elemento sostenedor de una pauta de comportamiento desadaptativo y autodestructivo. Esquemáticamente esta conceptualización se presenta de la siguiente manera.

\section{Esquema 5}

\section{Impacto Vital del paso por el COD}

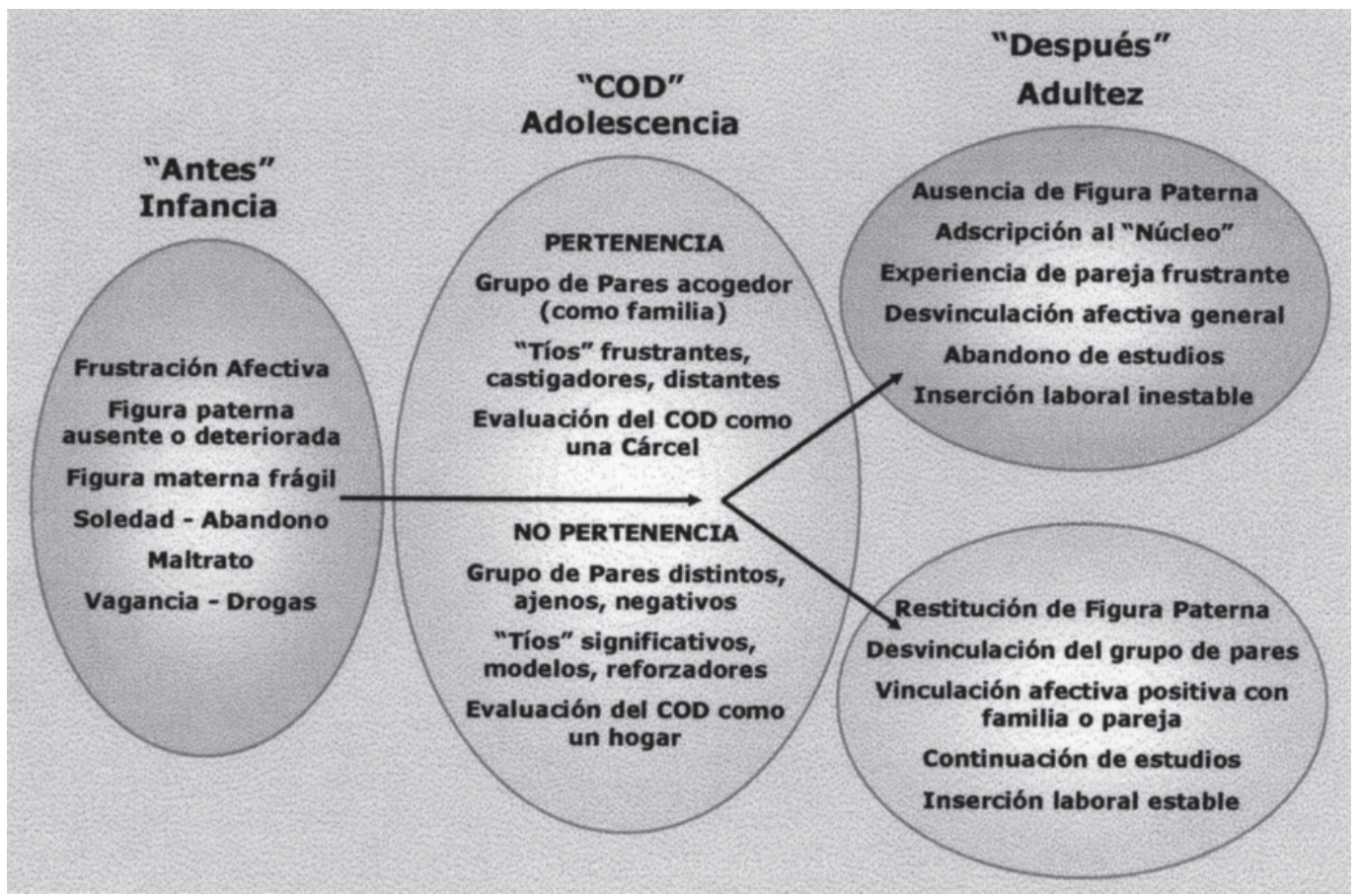




\section{Discusión}

Luego de una primera aproximación hacia las vivencias individuales de los participantes, se observa que las experiencias familiares tempranas están marcadas por relaciones vinculares que se han descrito teóricamente para las familias en situación de pobreza y marginalidad (Pérez-Luco, 1994; Zambrano, 2001). Los sujetos dan a conocer que en épocas de infancia y adolescencia el vínculo más conflictivo es con la figura paterna (padre biológico o padrastro), pues quienes asumen este rol muestran frecuentes conductas de consumo excesivo de alcohol, agresividad y despreocupación hacia los hijos y esposas, alterando la formación de vínculos afectivos sanos o en otros casos tornando éstos en una relación conflictiva o de rechazo (Barudy, 2001). Simultáneamente, la experiencia familiar se encuentra marcada y sostenida por la formación de fuertes vínculos afectivos positivos, con las madres, las que asumen los roles de cuidado, protección y afectividad explícita, y también con los hermanos, a los que consideran parte del endogrupo, potenciando la identificación con éstos. Relevante es el salto transgeneracional que hay en la formación de vínculos afectivos positivos entre nietos y abuelos (Boszormenyi-Nagy y Spark, 1983), siendo considerados estos últimos como figuras de apoyo positivas, reparadoras de los vínculos dañados o perdidos con los padres y como medio de apoyo y protección auxiliar.

La teoría nos muestra que en familias marginales se da que los padres con desarrollo emocional interferido por enfrentamiento a experiencias límites tempranas tales como pérdidas afectivas, abandono, encarcelamiento, búsqueda de subsistencia, etc.; mantienen dinámicas conflictivas al constituir sus vínculos familiares, especialmente con los hijos, resultando una perpetuación de relaciones de carencias afectivas a nivel transgeneracional (Pérez-Luco y Alarcón, 1992; Rodríguez, 1989). Esto se da tanto por una condición de sobrevivencia a lo largo de una vida difícil de soportar por los sujetos (experiencia crónica), unido a consecuencias psicológicas que llevan a actos y conductas autodestructivas (Ávalos, 1990; Fischman, 1989; Rubio, 1985;). Así vemos que los participantes tienden a repetir la pauta familiar debido fundamentalmente a dos motivos: por una parte, su experiencia infantil con la figura paterna; y por otra, la insatisfacción permanente de necesidades básicas que no han sido resueltas en el transcurso de su vida adulta, por lo que al enfrentar la paternidad, los sujetos inconscientemente actualizan sus frustraciones, propiciándose un círculo vicioso transgeneracional.

En cuanto a los vínculos afectivos positivos establecidos con las madres, igual ocurren en un contexto disfuncional, pues ellas también presentan un desarrollo emocional deficiente por experiencias tempranas similares, traspasándoles a sus hijos contenidos vivenciales negativos propios, como traumas personales, (Biedermann y Díaz, 1991). Esto dificultaría la individuación y el establecimiento de relaciones de pareja sanas y estables en la adultez, especialmente en los hombres, como se observa en el estudio, lo que se comprende en función de una temprana pérdida de la posición de centralidad en la familia, asumiendo, por su condición de género (masculino) un doble rol, el de proveedor y de gratificador de necesidades afectivas de la madre, roles que deben asumir los hijos por no cumplirlos sus parejas.

Los sujetos refieren comportamientos desadaptativos en su infancia, como consumo de sustancias, hurtos e inadaptación escolar. La teoría de la desadaptación social señala con alta frecuencia trayectorias escolares deficitarias, manifestadas en escolaridad básica incompleta, con escasa probabilidad de estudios técnicos y menos superiores (Fuenzalida, 1996). Sin embargo en uno de los casos presenta estudios secundarios completos, lo que coincide con la ausencia de 
conflictos con la justicia en su vida adulta no presentando una activa carrera delictiva. La deserción escolar en estos casos se basaba frecuentemente en la necesidad de generar recursos para cooperar con el sustento económico familiar y además por la atracción del trabajo como actividad que garantiza independencia y gratificación de las necesidades más apremiantes, como comida, vestimenta y ocio. En otros casos la deserción escolar se gatilla por la iniciativa de "moverse en la calle", debido a la percepción de ésta como enriquecedora y motivante, al brindar la sensación de libertad para ser y actuar, observándose que la materialización de actos delictivos es evaluada como un medio de realización personal, pues garantiza la satisfacción de necesidades de forma inmediata y más accesible, hechos acogidos y validados en el grupo de pares encontrado en la calle.

El grupo de la calle, como todo grupo tiene sus propias reglas y dinámicas para garantizar la pertenencia, satisfaciendo necesidades gregarias de identidad y protección; y permitiendo la sublimación de tensiones psicológicas; se demuestra la fuerte influencia de los "pares", especialmente cuando el grupo presenta pautas de comportamiento antisocial (Urra, 2000; Valverde, 1996). Los cuatro participantes del estudio explícitamente refieren su incorporación a grupos de la calle como consecuencia de dificultades familiares y la comisión de los primeros actos delictivos para conseguir la pertenencia y aprobación del grupo. Así la pandilla es asumida como familia sustituta que proporciona apoyo emocional, entretención y sensaciones nuevas, en un marco de libertad sin restricciones.

Si bien la deserción escolar es percibida por los sujetos como una resolución personal adecuada, debido a las garantías de la calle y los grupo o "pandillas", la teoría muestra que estos vacíos educativos tienen implicancias en la construcción de identidad, ya que el niño al tener dificultades para presentar las conductas esperadas para su edad y para interactuar apropiadamente con los otros, experimentará la tensión de no responder a las expectativas sociales. Esto redunda a nivel psicológico en una baja autoestima, sentimientos de frustración y surgimiento de mecanismos psicológicos que permitan compensar las dificultades experimentadas en la relación con los otros (Moreno, 2001; Valverde, 1996).

Como afirma Valverde (1996) se perfilaría en la vida de los sujetos condiciones adversas de existencia que imposibilitan el desarrollo de cierto tipo de recursos exigidos por el medio social, potenciando de esta manera el proceso de inadaptación social. Así, la inadaptación al fin y al cabo resulta ser una forma de adaptación difícil a condiciones de vida insatisfactorias, pues deben desarrollar estrategias alternativas para responder a las múltiples demandas de un medio que no ofrece oportunidades. Las condiciones de vida precarias, en lo material y lo afectivo, imposibilitan a los niños que allí se desarrollan aprender oportunamente normas, formas de relación, habilidades y competencias que le permitan integrarse adecuadamente a la vida social (Zambrano, 2001).

De acuerdo a lo anterior, a pesar de las iniciativas personales de los jóvenes, las oportunidades son restringidas y mezquinas para lograr una adecuada integración social, favoreciendo la mantención de pautas de conductas desadaptadas personal, social y legalmente y potenciando la continuidad de la carrera delictiva hacia la adultez. Esto es evidente en algunos de los casos que expresan haber asumido explícitamente su actividad delictiva como trabajo y forma de vida válida en su entorno social.

Dentro de los aspectos más relevantes que arrojan los contenidos de las distintas entrevistas realizadas a los sujetos, surgen con mucha fuerza las experiencias vividas durante el periodo de internamiento en el COD, lo que permite comprender de mejor manera 
las repercusiones que tuvo en los sujetos, y como influyó en mayor o menor medida en sus vidas y en las distintas direcciones y manifestaciones que ella ha tomado.

Las vivencias internas dentro del COD, por lo general apuntan a la percepción de las personas con las que se tuvo relación: los funcionarios y los compañeros. En esto se puede apreciar una cierta categorización de los grupos de sujetos, lo que se relaciona con la teoría de la identidad social (Tafjel y Turner, 1979), la cual plantea que la categorización permite al individuo definirse como miembro de grupos específicos en el seno de la estructura social, en nuestro caso estructura emanada desde el propio centro (COD). La teoría agrega que como resultado de esta categorización, el individuo acaba identificándose con ciertos grupos particulares similares en sexo, edad, etnia, clase social, etc., lo que nos explica en mejor medida la cohesión grupal relativa entre pares en el COD, puesto que comparten por lo general todas las características antes mencionadas. Esta diferenciación entre endogrupo y exogrupo, influye desde nuestro punto de vista en la percepción de los chicos hacia los tíos, sobretodo en la primera etapa seguida al ingreso. Luego dependiendo del tiempo de permanencia, existiría un periodo de habituación y mayor acceso al exogrupo en este caso representado por tíos y funcionarios, lo que llevaría a estrechar lazos en busca de figuras adultas significativas, dado la ausencia de imágenes parentales cercanas. Las percepciones respecto de estos funcionarios varían, de acuerdo a características personales y acciones de los mismos, siendo recurrente la percepción de abusadores debido a experiencias de maltrato físico, castigo injusto, trabajo forzado e incluso el patrocinar abuso sexual, pero también la percepción positiva de brindar apoyo siendo percibidos como acogedores y en algunos casos promotores de un trato preferencial hacia ellos.

En relación a la percepción del grupo de pares, en gran medida se les ve conflictivos, por experiencias de agresión y rechazo, pero también como amigos, dado que entre pares se da una relación de compañerismo con grupos específicos (compañeros más cercanos o de la misma pandilla). La teoría antes mencionada plantea que la identidad social sería parte del concepto de sí mismo de un individuo que se halla ligada al conocimiento de su pertenencia a ciertos grupos sociales y a la significación emocional y evaluación que resulta de esta pertenencia (Tafjel y Turner, 1979). Es por esto que los sujetos dentro del COD tienen la tendencia a formar grupos, lo que en cierta medida les daría el soporte emocional y la seguridad que dentro de ese centro carecen. Se hipotetiza que el gran número de relaciones conflictivas entre pares, se puede atribuir a la desadaptación que sufre el sujeto al insertarse a este centro, pues se le exige al joven institucionalizado ser autónomo y al mismo tiempo, someterse a reglas impersonales, externas y preexistentes, a las cuales los sujetos desde nuestra perspectiva no logran adaptarse por no poseer en muchos casos los recursos y las estrategias de enfrentamiento adecuadas, para este tipo de interacción interpersonal, carencias que en gran medida vienen dadas por el tipo de pautas de relación deficientes otorgadas por las familias de origen. Algunos investigadores señalan que en este tipo de situación los vínculos con los coetáneos, se convierten en el único factor favorecedor de la evolución moral, lo que explica en cierto modo, la coexistencia de relaciones de compañerismo entre pares, pese a los conflictos que se generan diariamente entre ellos (Egenau y Nicholls, 1990). Cabe señalar que, en relación a las experiencias interpersonales, las acciones llevadas a cabo con los pares muchas veces se relacionaban con la agresión entre estos mismos, pero también en gran medida se generaban acciones de cooperación y de tipo lúdicas, como deportes y juegos, entre ellos. Una importante regularidad arrojada por los relatos, es que los sujetos que se identificaron con los pares al interior del COD 
siguieron el camino delictivo y aquellos en los que no existió tal identificación, no continuaron en actividades delictivas posteriores a la estadía en el COD.

En relación con los sentimientos evocados por los sujetos al recordar el período de internación, en su mayoría se refieren emociones de tipo negativo como impotencia, soledad, engaño y sentimientos de encierro, siendo esta última preponderante en sus relatos. Muchos de estos sentimientos pueden estar dados por una inadaptación subjetiva, la cual se manifiesta cuando las instituciones correctoras y protectoras no responden de forma adecuada a las necesidades del sujeto, profundizándose el conflicto y produciendo alteraciones en el comportamiento y la personalidad del inadaptado, como consecuencia del proceso de internación (Valverde, 1996).

Estos sentimientos en su mayoría negativos y aversivos para la integridad psíquica de los sujetos se ven potenciados por una desestimación personal, la cual desde la teoría puede explicarse como una consecuencia lógica del mirar hacia fuera y verse ajeno, y a su vez mirase hacia adentro y no encontrar nada de valor (Pérez-Luco, Alarcón y Lucero, 1992). Los sujetos expresan haberse sentido marginales, inadecuados, malos y desadaptados. Su historia les demostraba su falta de valor y su paso por el COD se los confirmó dado el trato abusivo y despersonalizado y el abandono experimentado. A lo anterior se suma la traumática experiencia de detención, vinculándose a la sociedad mayor a través de los organismos policiales y el sistema judicial, lo que fue vivenciado como el estar siendo procesado por un delito. Aunque para los profesionales del sistema el ingreso al COD constituya una medida de protección, para los participantes del estudio aquélla experiencia significó la etiquetación como delincuentes que recibían el castigo merecido por sus faltas, por tanto, la permanencia en el centro con independencia de las causas, significó una marca o estigma que nunca más se ha borrado, con el previsible impacto que ello tiene en el desarrollo posterior de sus identidades (Goffman, 1998). Este impacto se hace evidente en el estudio, pues dos de los sujetos explícitamente refieren haber asumido una identidad delictiva a partir de esa época y los otros dos resienten la estigmatización sufrida como consecuencia de su paso por el COD, marca contra la que han luchado permanentemente sin poder deshacerse de ella.

El egreso del COD también resulta conflictivo, pues sólo uno de los participantes fue retirado finalmente por la familia luego de una larga estadía, estadía que sin embargo no fue continua, pues en una ocasión se fugó y fue recapturado, por lo que tuvo un segundo ingreso. Los otros tres participantes tuvieron frecuentes ingresos en un lapso de aproximadamente dos años, siendo la mayoría de sus egresos por fuga. A partir de los relatos se hipotetiza que la fuga cumplía la función de mitigar momentáneamente los sentimientos de encierro y soledad, pero también era un modo de demostrar descontento frente al trato y reglas impuestas; sin embargo, pese a lo anterior, el COD se percibía como refugio material y afectivo frente a las precarias condiciones de vida y a los reiterados conflictos familiares, por lo que, luego de lo traumático del primer ingreso, ser recapturado y volver resultaba "deseable", pues era un lugar "familiar" en el que se encontraban seguros, eran reconocidos e incluso "bienvenidos".

La teoría nos dice que la "retirada" es un medio que sirve de refugio en conductas y estilos de vida que tienden a evitar el enfrentamiento y la participación en la sociedad. La fuga en este caso supone una solución al conflicto individuo-sociedad, expresado en conductas y actitudes antisociales o de enfrentamiento, violencia y ruptura con las normas, De acuerdo a los relatos, las fugas se producían regularmente en grupos y lue- 
go de periodos en que la tensión interna había aumentado hasta "explotar" en agresiones directas entre pares o entre los adolescentes y los tíos (en una u otra dirección), cumpliendo de este modo la función de "válvula de escape" circunstancial. Se entiende así que las fugas, como causal de egreso del COD, cumple una función reafirmadora de la identidad delictiva, anticipando la experiencia de episodios de libertad y reclusión.

En los participantes que no siguieron el camino delictivo se percibe el período de permanencia en el COD como una fuente de privación de oportunidades para compartir con la familia o continuar estudios, siendo lo más negativo el ser privados de su libertad. Los participantes que continuaron delinquiendo, si bien también realizan una evaluación más negativa que positiva, consideran la experiencia «inútil», pues no les aportó las herramientas necesarias para superar su condición ni mejorar su calidad de vida.

Esta evaluación retrospectiva, contextualizada en los relatos de los participantes, nos da luces respecto del impacto vivencial de la experiencia, pues la calidad de los vínculos afectivos previos al ingreso y la referencia a figuras masculinas adultas es claramente distinta entre quienes asumen una identidad delictiva y quienes no lo hacen, lo que además se asocia directamente a la calidad de los vínculos afectivos establecidos con posterioridad.

Quienes continúan delinquiendo relatan una mayor traumatización en sus experiencias infantiles y ausencia total de vínculos positivos con figuras masculinas adultas, relatando mayores conflictos con los tíos (autoridad) durante su internación, identificación con el grupo de pares y fracaso reiterado en la constitución posterior de una familia; se entiende la inutilidad de la medida en tanto no les permite resignificar su experiencia previa ni construir a partir de ello vínculos de mejor calidad, incrementándose los sen- timientos negativos hacia el mundo y la realidad que les toca vivir ("resentimiento social") y avalando, en consecuencia, la opción por la delincuencia como forma de vida.

Quienes no continuaron delinquiendo, si bien también hablan de mucha precariedad material, insatisfacción, frustraciones y pérdidas afectivas importantes, rescatan en sus experiencias previas la existencia de algunos vínculos familiares positivos y la presencia de figuras masculinas positivas que actúan como modelos, refiriendo relaciones más positivas con los tíos, rechazo hacia el grupo de pares en el COD y mayor éxito en la constitución de la propia familia; se entiende así la valoración de la experiencia como "castigo ejemplificador", en tanto permite una redefinición de la propia conducta optando por la adaptación social.

Si bien para todos los participantes la condición de pobreza material se ha mantenido, la experiencia laboral ha sido poco gratificante siempre y ninguno se ha propuesto metas personales para su futuro, manifestando sueños incontrolables para ellos (ganarse el kino) como expectativa de mejoramiento de su condición actual; la diferencia evidente está en la calidad de los vínculos afectivos experimentados a lo largo de su vida y en especial en la presencia de modelos masculinos adultos significativos durante la adolescencia. Estos dos hechos que constituyen datos no observados en la literatura científica revisada, nos llevan a resaltar la relevancia de los vínculos afectivos como dimensión determinante de la adopción de una identidad delictiva durante esta etapa, enfatizando la necesidad de orientar el trabajo de profesionales y técnicos de los actuales CTD hacia una modelación basada en la formación de vínculos estrechos entre adolescentes y tutores especialmente capacitados para ello.

La hipótesis de la vinculación, si bien también ha sido resaltada previamente como estrategia de intervención (Alarcón, Pérez-Luco 
y Lucero, 1992), adquiere en este caso una dimensión distinta en cuanto se aprecia la relevancia otorgada por los participantes del estudio a la experiencia de internación en el COD. Esto dado que nos enfrentamos en la práctica a la sistemática ocurrencia de un efecto negativo de las "medidas de protección" implementadas, pudiendo sostenerse que "dividen aguas" contribuyendo en la mitad de los casos a la consolidación de una identidad delictiva.

En consecuencia, el postulado sostenido deriva en la necesidad de profesionalizar la función de los educadores de estos centros, formando en ellos las competencias necesarias para asumir la labor de "modelos de adaptación social" que cuenten con las herramientas técnicas de observación e intervención apropiadas para favorecer el desarrollo de vínculos positivos que favorezcan una intencionada resignificación de la experiencia previa, bastante generalizada entre los jóvenes que ingresan a los centros y profusamente descrita en la literatura científica. Creemos que asumir este desafío podría significar un mejor impacto en la prevención de la delincuencia y probablemente un menor costo en el largo plazo.

\section{Referencias Bibliográficas}

Alarcón, M.; Alongueo, P.; Bandet, J. Y VERGARA, E. (1992). El rol de la escuela en el proceso de formación de identidad en jóvenes urbano-populares de la ciudad de Temuco. Tesis de Licenciatura en Psicología. Temuco: Universidad de La Frontera.

Alarcón, P.; Pérez-Luco, R. Y Lucero, C. (1992). Perfil emocional de los niños que delinquen como estrategia de adaptación: una comprensión clínico-social como propuesta de trabajo. Temuco: Revista Frontera $\mathrm{N}^{\mathrm{o}} 11$ (9-18).

Alarcón, M. Y Troncoso, E. (2001) Familia: Redes y vínculos para el desarrollo. En R. Pérez-Luco (Comp.). De la mano de los niños: nuevas miradas para construir nuevos caminos. Temuco: UFRO-SENAME.

Ávalos, S. (1990). Los menores infractores. Niños de la calle. Santiago: UNICEF-Hogar de Cristo.

BARUdy, J. (2001). Maltrato infantil. Santiago: Editorial Galdoc.

Biedermann, N. Y Díaz, M. (1991). Detenidos desaparecidos en Chile: Consecuencias para la segunda generación. Santiago: ILAS.

BOSZORMENYI-NAGY, I. Y SPARK, G. (1983). Lealtades invisibles. Buenos Aires, Amorrortu.

Conejeros, L. Y Torres, L. (1992). Prostitución infanto-juvenil en la ciudad de Temuco: un enfoque psicosocial. Tesis de Licenciatura en Psicología. Temuco: Universidad de La Frontera.

Cooper, D. (1994). Delincuencia común en Chile. Santiago: LOM Ediciones.

Egenau, P. Y Nicholls, E. (1990). Juventud, delincuencia y prisionización. En Generación (Comp.) Los jóvenes en Chile hoy: Santiago: Cide-Cieplan-Inchi-Psi-Sur.

Fischman, CH. (1989). Tratamiento de adolescentes con problemas. Capítulo III: Tratamiento de la delincuencia: Abordar las premisas del sí mismo. Buenos Aires: Paidós.

FuENZALIDA, I. (1996). El medio social desventajoso y la conducta socialmente desviada. Revista Chilena de Ciencia Penitenciaria $y$ de Derecho Penal. No 22, (13-19).

Goffman, E. (1998). Estigma. La identidad deteriorada. Buenos Aires: Amorrortu.

LlanOS, M. Y Sinclair, A. (1991). La familia del menor infractor de ley. Revista chilena de ciencia penitenciaria y derecho penal. $\mathrm{N}^{\mathrm{O}}$ 16 (123-129). 
Martínez, C.; Neculhueque, G.; Oliveros, I. Y Sото, А. (2003). Estrategias de afrontamiento ante el estrés psicosocial en familias que viven en condición de pobreza en la ciudad de Temuco. Tesis de Licenciatura en Psicología. Temuco: Universidad de La Frontera.

Moreno, M. (2001). Psicología de la marginación social. Concepto, ámbito y actuaciones. Málaga: Ediciones Aljibe.

Oñate, X. Y Calfuguir, M. E. (1998). Estudio exploratorio inferencial de las condiciones de pobreza y potencial de desarrollo en familias de cuatro comunas de la Región de La Araucanía: Una tipología empírica. Tesis de Licenciatura en Psicología. Temuco: Universidad de La Frontera.

Paz Ciudadana (2003). Informe de seguridad ciudadana y políticas públicas. Santiago: Fundación Paz Ciudadana.

Pérez-Luco, R. (1994). Pobreza en América Latina: significados y abordajes. Pasos hacia una visión ecosistémica. Tesis de Maestría. México: FLACSO

Pérez-Luco, R. (2003). Análisis de Contenidos Jerárquico Ponderado. Curso Metodología Cualitativa Avanzada: Magíster en Psicología. Temuco: Universidad de la Frontera.

Pérez-Luco, R. Y Alarcón, P. (1992). Impactos psicosocial de la condición de opresión permanente en los sectores marginales. Temuco: Revista Frontera $\mathrm{N}^{\circ} 11$ (55-60)

Pérez-luco, R.; Alarcón, P. Y Zambrano, A. (2004). Desarrollo Humano: Paradoja de la estabilidad del cambio, breve análisis desde la Psicología. Revista Intervención Psicosocial. Vol. 13, No 1, (39-61)

Rodríguez, C. (1989). Cicatrices de la pobreza. Un estudio psicoanalítico. Caracas: Nueva Sociedad.

RuBIO, V. (1985). Estudio de carencias afectivas y la posible influencia negativa futura. Ma- drid: Dirección General de Protección Jurídica del Menor, Ministerio de Justicia.

Sepúlveda, E. Y Contreras, R. (1985). Calidad de vida del menor en situación irregular. Sus implicaciones vivenciales. Santiago: Primer Congreso Nacional de Psicólogos.

Tafjel, H. Y Turner, J. C. (1979). An integrative theory of intergroup conflict. In W.G. Austin y S. Worchel (Eds.). The social psychology of intergroup relation. Belmos, CA: Wadsworth.

TAYLOR, S. Y BOGDAN, R. (1986). Introducción a los métodos cualitativos de investigación. Buenos Aires: Paidós.

URRA, J. (2000). Adolescentes en conflicto. 29 casos reales. Madrid: Pirámide.

Valverde, J. (1996). Proceso de inadaptación social. Madrid: Editorial Popular S.A.

VERGARA, R. (1996). Ladrones y sociedad: análisis cultural de su dinámica. Revista chilena de ciencia Penitenciaria y derecho penal. $\mathrm{N}^{\mathrm{o}} 22$.

Zambrano, A. (2001). Pobreza, marginalidad y desviación: el rol mediador de la identidad familiar. En R. Pérez-Luco (Comp.). De la mano de los niños: nuevas miradas para construir nuevos caminos. Temuco: UFROSENAME.

Fecha Recepción Artículo: 28 de junio 2005 Fecha Evaluación Final: 10 de abril 2006 
